\title{
Solvent-Dependent Light-Induced Structures in Gem-Dichlorocyclopropanated Polybutadiene Solutions
}

\author{
A. Bogris, ${ }^{\dagger, \ddagger \odot ~ J . ~ W a n g, ~}{ }^{\S \odot}$ M. Anyfantakis, ${ }^{\| \odot}$ B. Loppinet, ${ }^{*}{ }^{\dagger} \odot$ S. L. Craig, ${ }^{\perp \odot}$ H-J. Butt, ${ }^{\# \odot}$ \\ and G. Fytas $*,+, \#$ \\ ${ }^{\dagger}$ Institute of Electronic Structure and Laser, Foundation for Research and Technology - Hellas, 70013 Heraklion, Crete, Greece \\ ${ }^{\ddagger}$ Department of Materials Science and Technology, University of Crete, 70013 Heraklion, Crete, Greece \\ ${ }^{\S}$ Department of Chemistry, Massachusetts Institute of Technology, 77 Massachusetts Avenue, Cambridge, Massachusetts 02139, \\ United States \\ "Physics \& Materials Science Research Unit, University of Luxembourg, 162a Avenue de la Faiencerie, Luxembourg L-1511, \\ Luxembourg \\ ${ }^{\perp}$ Department of Chemistry, Duke University, Durham, North Carolina 27708, United States \\ \#Max-Planck Institute for Polymer Research, 55128 Mainz, Germany
}

Supporting Information

\begin{abstract}
The formation of permanent structures upon mild red laser illumination in transparent polydiene solutions is examined in the case of gem-dichlorocyclopropanated polybutadiene ( $g \mathrm{DCC}-\mathrm{PB})$ polymers bearing $15 \%$ functional units of the dichlorocyclopropane groups. The response was found to be distinct from the precursor PB. Whereas fiber-like patterns were clearly observed in both precursor and $g \mathrm{DCC}-\mathrm{PB}$ solutions in cyclohexane, these were absent in the case of $g \mathrm{DCC}-\mathrm{PB} /$ chloroform but were present in the precursor $\mathrm{PB} /$ chloroform solutions. The involved mechanical stresses were not sufficient for the $g \mathrm{DCC}$ activation to be detected by NMR spectroscopy. Remarkably, addition of even 10 wt $\% g D C C-P B$ into the latter solution sufficed to suppress

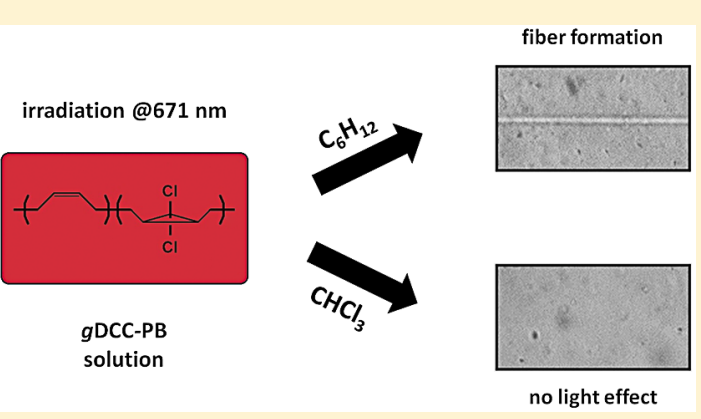
the light-induced patterning. The importance of the chemical environment on the response to light irradiation was further checked and confirmed by use of other PB copolymers. Different diameter patterns and kinetics were observed. The strong solvent and comonomer mediated effect was reflected neither in solvency nor in optical polarizability differences of the polymers solvent couples.
\end{abstract}

\section{INTRODUCTION}

A wide range of physical properties of polymers is directly influenced by their microstructure, such as the structural isomerism that is in polymers with double bonds along their backbone. In the case of copolymers, the fraction and the sequence of the two different monomers define both the properties and the response to external stimuli. It has been known since the 19th century that mechanical forces (e.g., milling, sonication, tension) can trigger chemical reactions distinct from the reactions induced by heat. ${ }^{1}$ Also, knowledge of mechanical response by light, through photochemical effects, such as photoactuation in soft systems, is advanced. ${ }^{2-4}$ In recent years, functional groups (mechanophores) with relatively weak covalent bonds that can change the fluorescent emission or become luminescent upon mechanically induced bond scission have been synthesized. ${ }^{5-8}$ This strain-induced optical response has been therefore utilized as a sensitive indicator of the stress-softening and toughening behavior of different polymer networks. ${ }^{9,10}$

Light of any frequency can also exert, albeit weak, forces on illuminated surfaces, as predicted by Maxwell. ${ }^{11}$ After the advent of lasers, the application of optical forces to manipulate ${ }^{12}$ and deform materials ${ }^{13,14}$ led to the new field of optomechanics. ${ }^{15}$ So far, it is restricted to hard dielectrics, whereas the optomechanics of soft materials (polymers and colloids) is essentially unexplored. ${ }^{16}$ Given the ease of deformability and the readily available plethora of functionalities, the potential of soft dielectrics is large. ${ }^{17-19}$ It is conceivable that light-induced matter deformation can activate the mechanophore action in the absence of external mechanical stress. In fully transparent viscoelastic polydiene solutions, a peculiar light effect not conforming to known types of electrostric$\operatorname{tion}^{11,18}$ led to matter association along the laser beam. ${ }^{20-24}$ The study of this effect has shown clearly the important role of the microstructure on the patterning efficiency and, in particular, the crucial role of 1,4-dienes. In the case of block copolymers, the required condition was established to be that one of the blocks should be polyisoprene (PI) or polybutadiene (PB). ${ }^{20}$

In this paper, we examine the possibility of light-driven postsynthetic modification in butadiene-based copolymers bearing

Received: March 13, 2018

Revised: June 6, 2018

Published: June 11, 2018 
Scheme 1. Structure of Gem-Dichlorocyclopropanated PB (gDCC-PB) ${ }^{a}$

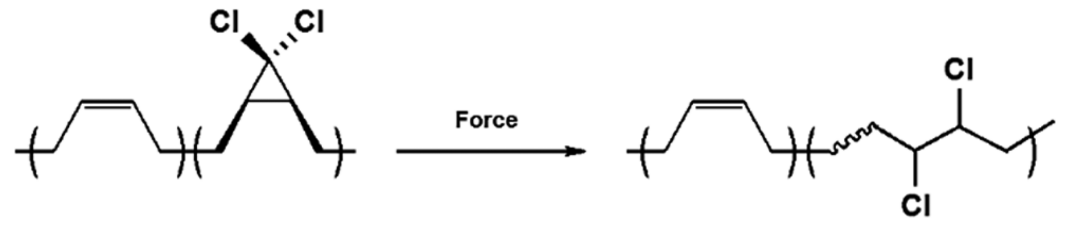

${ }^{a_{T}}$ The application of mechanical force to the $g$ polymer subchain accelerates the ring opening of a dichlorocyclopropane to its corresponding 2,3-dichloroalkene. ${ }^{25}$.

mechanophore groups and the role of the solvent on the strength of the light-matter effect. In particular, we report on the interaction of cis-PB bearing about $15 \%$ covalently bonded gem-dichlorocyclopropane ( $\mathrm{gDCC}$ mechanophore $)^{25}$ in two good solvents (cyclohexane and chloroform) with light. We observed the formation of the fiber-like pattern in the $g \mathrm{DCC}-\mathrm{PB}$ copolymer (Scheme 1) solutions in cyclohexane, while ${ }^{1} \mathrm{H}$ NMR confirmed no ring opening of the mechanophores in the lightinduced $g \mathrm{DCC}-\mathrm{PB}$ network. For comparison, we used random copolymers with various butadiene cis-1,4 concentration along their backbone. The importance of the solvent regarding the kinetics of the pattern formation was revealed in the case of chloroform. In contrast to the $\mathrm{PB}$ precursor, the laser illumination has no discernible effect in the $\mathrm{gDCC}-\mathrm{PB} / \mathrm{CHCl}_{3}$ solutions, emphasizing the solvent impact on the light-induced pattern. $^{26}$

\section{EXPERIMENTAL SECTION}

Materials. The PB homopolymer was received from SigmaAldrich and was used as the precursor polymer for the synthesis of $g \mathrm{DCC}-\mathrm{PB} .^{6}$ This was specifically synthesized to contain $15 \% g \mathrm{DCC}$ groups, randomly placed across the backbone of $\mathrm{PB}$. The styrene butadiene rubber (SBR) copolymer was received from Polimeri Europa (Eni S.p.A.), and both 1,2-PBs were purchased from Polymer Standards Service. The characteristic properties are listed in Table 1 . The solvents were purchased

Table 1. Polymers Molecular Characteristics

\begin{tabular}{llcccl}
\multicolumn{1}{c}{ polymer } & $\begin{array}{c}\text { code } \\
\text { name }\end{array}$ & $\begin{array}{c}M_{\mathrm{w}} \\
(\mathrm{g} / \mathrm{mol})\end{array}$ & $\begin{array}{c}\text { vinyl content } \\
(\%)\end{array}$ & $\begin{array}{c}1,4 \text { content } \\
(\%)\end{array}$ & $\begin{array}{c}\text { composition } \\
(\%)\end{array}$ \\
precursor PB & PB & $270 \mathrm{k}$ & & $>90$ & - \\
gDCC-PB & gPB & $300 \mathrm{k}$ & & 76 & gDCC: 15 \\
SBR & SBR & $162 \mathrm{k}$ & 66 & 12.9 & styrene: 21.1 \\
$1,2-\mathrm{PB}(1 \%)$ & PB1 & $317 \mathrm{k}$ & 99 & 1 & - \\
$1,2-\mathrm{PB}(30 \%)$ & PB30 & $88 \mathrm{k}$ & 70 & 30 & -
\end{tabular}

from Sigma-Aldrich and used as received. All polymer solutions in cyclohexane $\left(\mathrm{C}_{6} \mathrm{H}_{12}\right)$ and chloroform $\left(\mathrm{CHCl}_{3}\right)$ were prepared in ambient conditions, at a fixed polymer concentration (10 wt \%) but the lowest molecular weight 1,2-PB copolymer; a higher concentration (20 wt \%) was prepared in order to ensure similar semidilute solution conditions. The refractive indices of precursor $\mathrm{PB}$ and the two solvents measured by an Abbe refractometer amount to $n_{\mathrm{PB}}=1.522, n_{\mathrm{CHCl} 3}=1.446, n_{\mathrm{C} 6 \mathrm{H} 12}=$ 1.426 , and $n_{\mathrm{C} 10 \mathrm{H} 22}=1.411$. The refractive index increment $(\mathrm{d} n / \mathrm{d} c)$ in the two solvents (three dilute polymer concentrations between 0.08 and 0.34 wt \%) were measured with an interferometric technique at $632.8 \mathrm{~nm} .{ }^{27}$ For $g$ DCC-PB, $\mathrm{d} n / \mathrm{d} c$ decreased from $0.114 \mathrm{~cm}^{3} / \mathrm{g}$ in $\mathrm{C}_{6} \mathrm{H}_{12}$ to $0.09 \mathrm{~cm}^{3} / \mathrm{g}$ in $\mathrm{CHCl}_{3}$, whereas for the precursor $\mathrm{PB}, \mathrm{d} n / \mathrm{d} c$ increased from $0.093 \mathrm{~cm}^{3} / \mathrm{g}$ in $\mathrm{C}_{6} \mathrm{H}_{12}$ to $0.111 \mathrm{~cm}^{3} / \mathrm{g}$ in $\mathrm{CHCl}_{3}$ (Figure $\mathrm{S} 6$ ).
Characterization of Mechanophore and Precursor PB. The $g \mathrm{~PB}$ and $\mathrm{PB}$ samples were characterized by dynamic (DLS) and static (SLS) light scattering measurements in dilute solutions, performed on an ALV-5000 digital correlator equipped with an automated ALV goniometer and a $130 \mathrm{~mW} / 532 \mathrm{~nm}$ solid-state laser. The polarized light scattering intensity autocorrelation function $G(q, t)=\frac{\langle I(q, t) I(q, 0)\rangle}{|I(0)|^{2}}$ was recorded at different scattering wave vectors, $q=\frac{4 \pi n_{s}}{\lambda_{0}} \sin \frac{\theta}{2}$, with $n_{\mathrm{s}}$ being the refractive index of the solvent, $\lambda_{0}$ the wavelength of the incident laser light in vacuum, and $\theta$ the scattering angle. The decay of the relaxation function, $C(q, t)=[G(q, t)-1]^{1 / 2}$, in dilute solution is dominated by a single decay due to the polymer diffusion. However, the DLS experiment of both polymers in $\mathrm{CHCl}_{3}$ revealed two step decays. The fast one was attributed to the translational diffusion of the polymer chains. The slow decay was attributed to the presence of large aggregates in the solutions leading to a $q$-dependent light scattering intensity (Figure S4). The contribution of the slow process (intensity and dynamics) was found to be very similar in both polymer solutions, and it was not considered further. The intensity $I(q)$ of the fast process was found to be weakly $q$-dependent and helped assign it to the polymer chains. It could be used to estimate the molecular weight using $R_{\mathrm{vv}}(q)=a(q) R_{\mathrm{T}}\left[\frac{I(q)}{I_{\mathrm{T}}}\right]\left(\frac{n_{\mathrm{s}}}{n_{\mathrm{T}}}\right)^{2}$, where $a(q)=C(q, 0), R_{\mathrm{T}}(T)=2.78 \times 10^{-5} \mathrm{~cm}^{-1}, I_{\mathrm{T}}$ is the scattering of the standard (toluene) under the same conditions, and $n_{\mathrm{T}}$ its refractive index. The $q$ and $c$ dependence of $R_{\mathrm{vv}}(q, c)$ allows determination of the weight-averaged molecular weight $\left(M_{\mathrm{w}}\right)$ and the second virial coefficient $\left(A_{2}\right)$ from $\frac{H c}{R_{\mathrm{vv}}(q=0)}=\frac{1}{M_{\mathrm{w}}}+2 A_{2} c$, where $H=\frac{\left[2 \pi n\left(\frac{\mathrm{d} n}{\mathrm{dc}}\right)\right]^{2}}{N_{\mathrm{A}} \lambda_{0}^{4}}$, with $N_{\mathrm{A}}$ being Avogadro's number; $A_{2}$ represents the interpolymer interaction in the solvent. Hence, the molecular weight (Table 2) compares well with the gel permeation chromatography (GPC) values. This fast process is used to both estimate $A_{2}$ (Figure S5) and compute the hydrodynamic radius from the fast diffusion coefficient (Table 2).

The hydrodynamic radius pertaining to the fast process was determined as $R_{\mathrm{h}}=\frac{k T}{6 \pi \eta D}$, where $D$ is the translational chain diffusion, $k$ is the Boltzmann constant, and $\eta_{\mathrm{s}}$ is the solvent viscosity. The overlap concentration $c^{*}$, above which the polymer solution is in the semidilute regime, is estimated from $c^{*}=\frac{3 M_{\mathrm{w}}}{4 \pi N_{\mathrm{A}} R_{h}^{3}}$. The molecular characteristics $\left(R_{\mathrm{h}}, R_{\mathrm{g}}, A_{2}\right.$, and $\left.M_{\mathrm{w}}\right)$

Laser Irradiation and Visualization of the Formed Structures. The experimental setup, which was used in our experiments, is schematically shown in Figure 1. We used a Zeiss Axioskop 2 optical microscope, put in an up-right position to allow simultaneous irradiation and observation of the polymer solutions placed in standard spectroscopy quartz 
Table 2. Characteristic Physical Parameters Table of the Two Copolymers in $\mathrm{CHCl}_{3}$

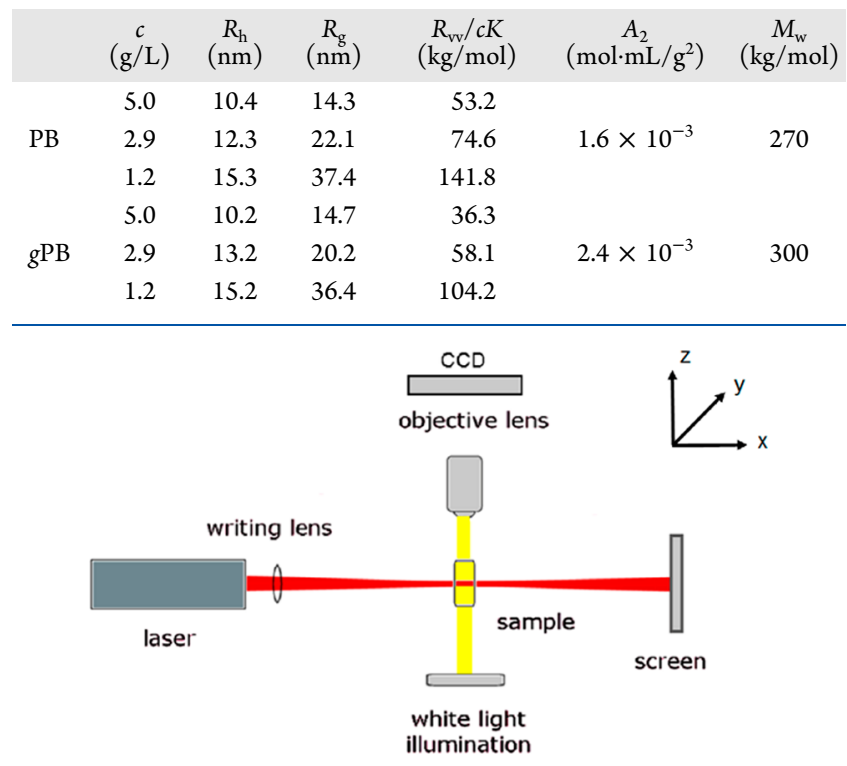

Figure 1. Schematic of the experimental setup. Samples are irradiated and simultaneously imaged under an optical microscope on the axis $(z)$ perpendicular to the laser beam along the $x$ direction. The transmitted laser beam is projected onto a screen.

cuvettes (Hellma, $4 \mathrm{~mm}$ path length). A DPSS cw laser (wavelength $671 \mathrm{~nm}$ and full power $\sim 60 \mathrm{~mW}$ ) was placed on the modified stage of the microscope. The laser beam was focused through a $4 \times(\mathrm{NA}=0.12)$ microscope objective on the entrance wall of the sample cell. The beam diameter at the focal point was about $20 \mu \mathrm{m}$ at the entry of the cell, and the transmitted beam spot exiting the cell was imaged on a paper screen.

For quantitative observation of the pattern formation, we used a variant of phase contrast microscopy. The technique provides a simple and general method to image refractive index inhomogeneities and it is particularly well adapted to the cylindrical geometry of the formed structures. The microscope Köhler illumination was used to produce a collimated white light beam impinging on the sample. The $5 \times$ microscope objective (Zeiss, NA $=0.15$ ) used for imaging was slightly defocused $(\sim 100 \mu \mathrm{m}$ above the focal plane on the $z$ axis). In such conditions, the intensity recorded on the CCD camera ( 8 bit, black and white) is related to the phase shift of the white light beam, which is induced by the refractive index difference between the formed pattern and the surrounding unperturbed solution. ${ }^{28-30}$

The imaged intensity is directly proportional to the second derivative of the phase shift induced by the formed structure, and the refractive index profile can be thus deduced. A local increase (decrease) of the refractive index will lead to a brighter (darker) stripe for positive defocusing (i.e., placing the focal plane above the structure). Assuming that the imaged local refractive index increase $\delta n$ can be attributed to the local polymer concentration $(\delta c)$, then the positive $\delta n$ corresponds to $\delta c>0$ in solutions with $\mathrm{d} n / \mathrm{d} c>0 .{ }^{23}$ Every image in a recorded time series was normalized by the first image $(t=0)$, and a normalized image intensity was computed, $I_{\mathrm{N}}(x, y, t)=\frac{I(x, y, t)}{I(x, y, 0)}-1$, where $I(x, y, t)$ is the intensity of an individual pixel at time $t$. The normalized intensity profiles across the pattern $(\Delta y \approx 60$ pixels) were first averaged over a portion of the image ( $\Delta x \approx 30$ lines) and were then integrated twice (with respect to the $y$ axis). To properly account for the different radii of the patterns, the intensity profile was then divided by the radius, and hence, the displayed profiles in Figure 2 are

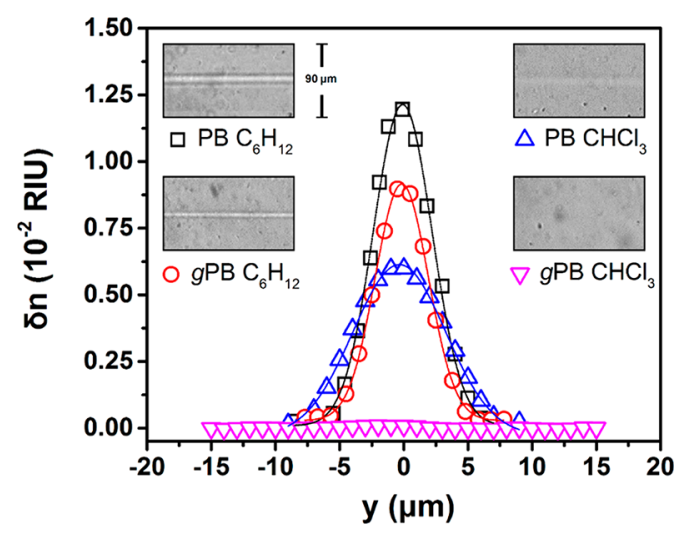

Figure 2. Phase contrast images (image size $169 \mu \mathrm{m} \times 90 \mu \mathrm{m}$ ) and corresponding refractive index profiles in $\mathrm{PB}$ and $g \mathrm{~PB}$ solutions in $\mathrm{C}_{6} \mathrm{H}_{12}\left(10\right.$ wt \%) and in $\mathrm{PB}$ and $g \mathrm{~PB}$ solutions in $\mathrm{CHCl}_{3}(10 \mathrm{wt} \%)$ after $300 \mathrm{~s}$ of laser illumination.

proportional to the refractive index profile. Moreover, the technique was calibrated using an optical fiber of known refractive index profile to obtain $\delta n$ in refractive index units (RIU). More details on the use of phase contrast microscopy are given in the Supporting Information. The pattern formation kinetics were determined by the evolution of the maximum normalized intensity profile $I^{*}$ (at the center of the pattern, $y=0$ ) with illumination time. ${ }^{31}$ The observed contrast relates to the refractive index gradient. It is worth noting that patterns in different samples were observed to have a different diameter. The overall refractive index increase (integrated over the pattern cross section) may then provide an alternative evaluation of the patterning kinetics, better adapted for comparison of pattern formation with different diameters (better than the refractive index gradient measured by $\left.I^{*}\right)$. This latter rate was obtained by measuring the rate of increase of $I^{*}$ and multiplying it by the square of the pattern radius (Figure S7).

\section{RESULTS}

Mechanophore and Precursor PB under Irradiation in $\mathrm{C}_{6} \mathrm{H}_{12}$ and $\mathrm{CHCl}_{3}$. The molecular characteristics of the two polymers and the thermodynamics of their dilute solutions in the two solvents are presented in the Experimental Section and in the Supporting Information (Figures S4 and S5). Both the $g \mathrm{~PB}$ copolymer and $\mathrm{PB}$ in $\mathrm{C}_{6} \mathrm{H}_{12}$ at $10 \mathrm{wt} \%$ polymer concentration, well above the overlap concentration $\left(c^{*}=0.18\right.$ wt \% in $\mathrm{CHCl}_{3}$ ), and at $20{ }^{\circ} \mathrm{C}$ exhibited the formation of fiber-like material patterns after light illumination (insets of Figure 2). In both solutions of $\mathrm{PB}$ and $g \mathrm{~PB}$ in $\mathrm{C}_{6} \mathrm{H}_{12}$, the light-induced patterns appeared qualitatively similar but displayed different refractive index contrasts $(\delta n(y=0))$, as seen in the different refractive index profiles of these images (Figure 2), which indicated weaker patterns in the $g \mathrm{~PB}$ than those in $\mathrm{PB}$ in $\mathrm{C}_{6} \mathrm{H}_{12}$ by about $25 \%$. The observed refractive index changes in the $\mathrm{PB} /$ $\mathrm{CHCl}_{3}$ solution after $300 \mathrm{~s}$ of irradiation are shown (blue triangles) in Figure 2. The pattern was noticeably broader than that in $\mathrm{C}_{6} \mathrm{H}_{12}$ solution, 9.5 compared to $5.7 \mu \mathrm{m}$. The refractive index contrast was more than twice lower in $\mathrm{CHCl}_{3}$ than that in $\mathrm{C}_{6} \mathrm{H}_{12}$. 
Remarkably, the writing effect was totally absent in the $g \mathrm{~PB} /$ $\mathrm{CHCl}_{3}$ solution (magenta triangles) even after prolonged illumination $(\sim 17 \mathrm{~h})$. The response of the two polymers to visible light illumination under identical conditions became dramatically different when $\mathrm{CHCl}_{3}$ was used as the solvent.

The "written" structures for both polymers (in $\mathrm{C}_{6} \mathrm{H}_{12}$ ) were permanent, in the sense that they did not fade out after cessation of the laser illumination. To monitor/detect a possible activation of mechanophore groups, we performed solution ${ }^{1} \mathrm{H}$ NMR, which is often used as a sensitive tool for evaluation of the ring opening ${ }^{25}$ of the mechanophore $g \mathrm{DCC}$, utilizing structures formed after prolonged irradiation (Figure S2). No evidence of ring opening (Figure S3) could be seen by NMR. This suggests that the irradiation did not produce stresses large enough for the ring opening.

Pattern Diameter and Kinetics of Different Copolymer Solvent Pairs. To further investigate the importance of the copolymer structure and composition on the observed pattern, we also used three additional random copolymers containing cis-1,4-butadiene: one SBR $(12.9 \%$ cis-1,4) and two 1,2-PB copolymers (30 and $1 \%$ cis-1,4), to be compared to the $g \mathrm{~PB}(76 \%$ cis-1,4) and PB (>90\% cis-1,4) precursor (see the Materials section, Table 1). We used the five copolymers dispersed in both $\mathrm{C}_{6} \mathrm{H}_{12}$ and $\mathrm{CHCl}_{3}$. Three of these copolymers (PB, PB1, and $\mathrm{PB} 30)$ were also dispersed in decane $\left(\mathrm{C}_{10} \mathrm{H}_{22}\right)$ for comparison; linear alkanes resulted in the fastest responses of $I^{*}{ }^{31}$ Noticeably, all irradiated solutions (with the noticeable exception

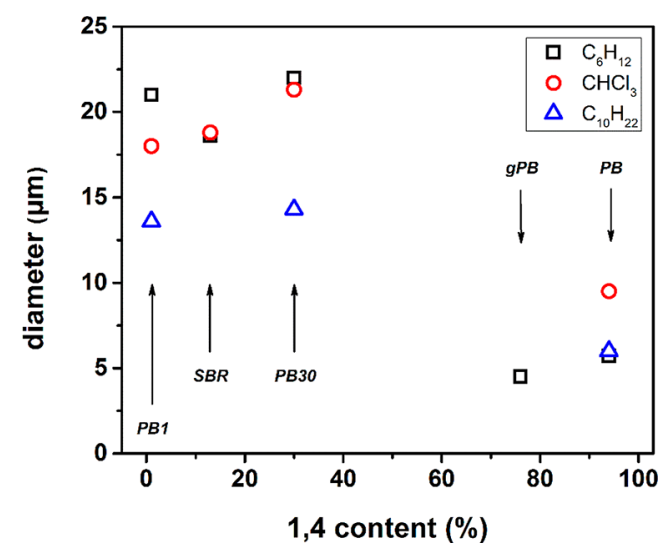

Figure 3. Diameters of the fibril patterns as a function of 1,4 content. of $g \mathrm{~PB}$ in $\mathrm{CHCl}_{3}$ ) responded to laser illumination by forming fibrillar patterns, though with different diameters and a broad range of kinetics. In an attempt to quantify the importance of the cis-1,4 content in the pattern formation and its kinetics, we report the diameters measured by phase contrast microscopy (Figure 3 ) and the estimated rates of formation as a function of the 1,4 content (Figure $4 b$ ).

For a given sample, the visible pattern did not change in diameter over time (within the resolution of the technique), a sign that the diameter was set early on in the process. The input laser beam had a fwhm (full width at half-maximum) of $\sim 20 \mu \mathrm{m}$ at the entrance of the sample cell. Pattern diameters were found to vary from sample to sample. The smaller diameters were around $5 \mu \mathrm{m}$, observed in alkanes for PB and $g \mathrm{~PB}$. The solutions in $\mathrm{CHCl}_{3}$ showed patterns with diameters larger than those of the alkane solvents, up to more than $20 \mu \mathrm{m}$ in PB1 and PB30. SBR patterns showed the same diameter in both $\mathrm{C}_{6} \mathrm{H}_{12}$ and $\mathrm{CHCl}_{3}$.

In all cases, the pattern fwhm was less than or equal to the beam fwhm. This is an indication of the locality of the process. A diameter lower than the fwhm reveals the self-focusing of the beam. The large difference of radius means that there was also a large difference of photon flux per unit area, up to a factor of $\sim 20$ between the narrower pattern $\left(\mathrm{gPB}\right.$ in $\left.\mathrm{C}_{6} \mathrm{H}_{12}\right)$ and the larger $\left(\mathrm{PB} 30\right.$ in $\left.\mathrm{CHCl}_{3}\right)$. The possible origins for the large differences are discussed below.

We evaluated the kinetics of the pattern evolution by measuring the image contrast $I^{*}$ vs $t$. As mentioned above, it measures the formation of refractive index gradients. The observed $I^{*}$ kinetics are displayed in Figure $4 \mathrm{a}$. $\mathrm{PB}$ and $g \mathrm{~PB}$ in $\mathrm{C}_{6} \mathrm{H}_{12}$ showed exponential-like growth, as previously reported for similar samples. ${ }^{31}$ The kinetics appeared to quickly reach saturation as $I^{*}$ reached a quasi-plateau after about $300 \mathrm{~s}$ under laser irradiation $\left(I^{*} \approx 0.20\right.$ corresponding to $\delta n \approx 10^{-2}$ RIU). Notably, this saturation was generally not observed in previous reports. ${ }^{31}$ The pattern formation in $\mathrm{CHCl}_{3}$ exhibited a qualitatively different type of kinetics with a more linear increase of $I^{*}$ with time for all copolymers. Noticeably, we did not observe any patterning for $g \mathrm{~PB}$ in $\mathrm{CHCl}_{3}$.

The kinetics of SBR solutions was also found to be linear, with very similar behavior in both solvents (diameter and contrast). The 1,2-PB copolymer solutions showed only very low contrast of the written pattern $\left(I^{*}<0.02\right)$; therefore, the type of kinetics observed was hard to establish.
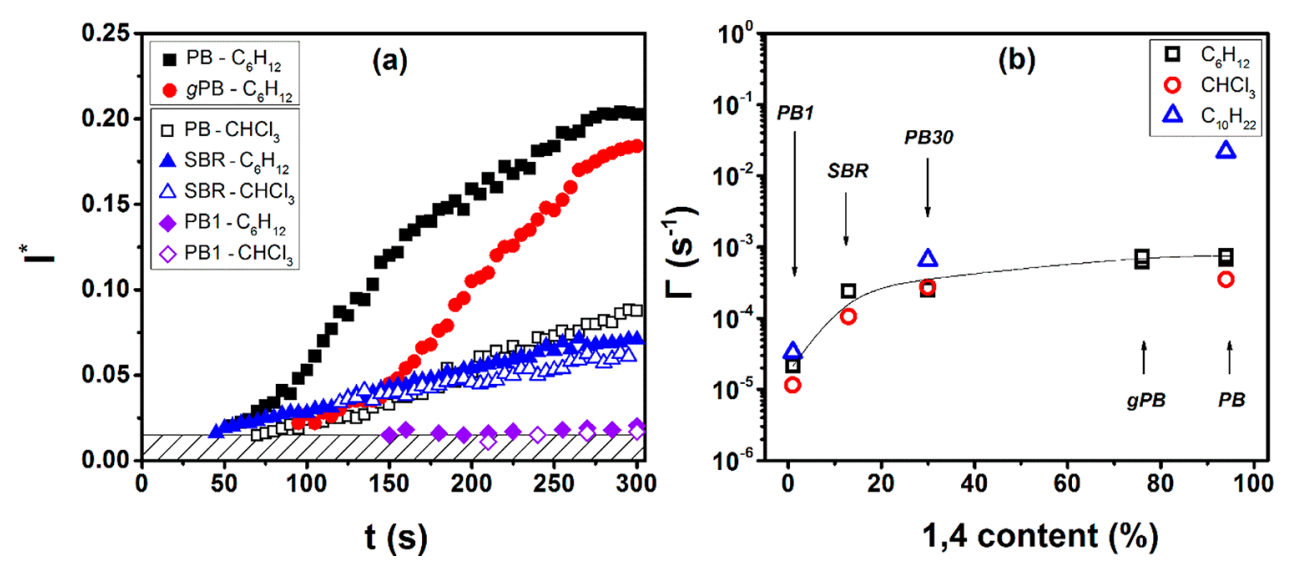

Figure 4. (a) Averaged normalized image intensity $\left(I^{*}\right)$ as a function of laser illumination time for $\mathrm{PB}, g \mathrm{~PB}, \mathrm{SBR}$, and $1,2-\mathrm{PB}$ solutions in $\mathrm{C}_{6} \mathrm{H}_{12}$ and $\mathrm{CHCl}_{3}$. (b) Dependence of the growth rate $\Gamma$ for formation of the structure on the 1,4 content of all of the used polymer solutions in $\mathrm{C}_{6} \mathrm{H}_{12}$, $\mathrm{CHCl}_{3}$, and $\mathrm{C}_{10} \mathrm{H}_{22}$. 
Characteristic growth rates of $I^{*}(t)$ were obtained as the slope of $I^{*}$ vs $t$ for the linear case (as a measure of the formation of the refractive index gradient $\delta n / a)$. For comparison, for the exponential-like kinetics of solutions in $\mathrm{C}_{6} \mathrm{H}_{12}$, a rate was calculated as the plateau value divided by time to reach the plateau (i.e., $300 \mathrm{~s}$ ). The characteristic rates are reported in Figure $4 \mathrm{~b}$ as a function of the 1,4 content. Solutions in $\mathrm{CHCl}_{3}$ exhibited a slower $I^{*}$ growth rate (red circles in Figure $4 \mathrm{~b}$ ) compared to the corresponding solutions in $\mathrm{C}_{6} \mathrm{H}_{12}$. All polymer solutions in $\mathrm{C}_{10} \mathrm{H}_{22}$ displayed the fastest growth rates (blue triangles in Figure 4b). Full 1,4-polymer in alkanes showed the faster formed patterns with a large refractive index gradient (large refractive index contrast and small dimension). The $I^{*}$ rate showed that the faster developing gradients are observed in a higher content of 1,4. Very low 1,4 content showed clearly much slowed down formation and a larger pattern. The amount of 1,4-monomer seems to affect the kinetics mostly in the very low amount regime (few \%). This is in qualitative agreement with little influence of the polymer (monomer) concentration on the kinetics. ${ }^{31}$ There is always an excess of 1,4 content, and this is not the rate-determining step.

Alternatively, we also report the time evolution of the integrated refractive index change, as characterized by $a^{2} I^{*}$ (Figures S7 and S8), where $a$ is the size (fwhm) of the refractive index pattern. Whereas $I^{*}$ characterizes the refractive index gradient that relates to optical properties, $a^{2} I^{*}$ provides a measure of the overall concentration evolution. The general conclusions as to the role of the 1,4 content in kinetics remain similar.

Mixtures of $g$ PB and PB. The above observations lead to the conclusion that the chemical environment is very important for the patterning effect. This importance of the chemical composition of the solutions is reinforced when going back to the specifics of $\mathrm{gPB} / \mathrm{CHCl}_{3}$ solution, where no patterning was observed. In order to address the remarkable impact of the comonomer-solvent effect, several mixtures of the mechanophore and precursor polymer were tested.

We increased the relative concentration of 1,4 active units by mixing the $g \mathrm{~PB}$ with its precursor homopolymer (PB) in different weight ratios (9:1,5:5, and 1:9), keeping the total polymer concentration in these solutions constant $(\sim 10 \mathrm{wt} \%)$. Surprisingly, for all ratios, we observed no pattern formation, even after $24 \mathrm{~h}$ of irradiation, as shown in Table 3; even $10 \%$ $g \mathrm{~PB}$ addition can inhibit the laser writing effect.

Table 3. Pattern Inhibition in Ternary $g \mathrm{~PB}$ and PB Solutions $\left(c=10\right.$ wt \%) in $\mathrm{CHCl}_{3}$ with 50 and $10 \% \mathrm{gPB}$ Composition

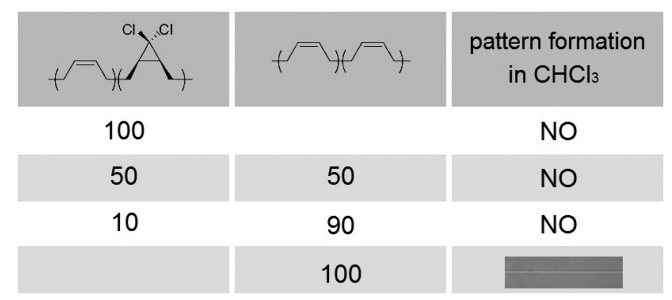

\section{DISCUSSION}

This latest observation is reminiscent of the cononsolvency according to which solvent mixtures become nonsolvents for a given polymer while both cosolvents are good solvents for this polymer. $^{26,32,33}$ In fact, $\mathrm{CHCl}_{3}$ is a good solvent for both the
$\mathrm{PB}$ and the $g \mathrm{~PB}$ based on the second virial coefficient $A_{2}$ and the radius of gyration $R_{\mathrm{g}}$ (Figure S5). The higher $A_{2}$ value (by about $40 \%$ ) of the $g \mathrm{~PB}$ solution implies stronger interchain repulsion than that for the $\mathrm{PB}$ precursor chains, and notably, it follows the polarizability trend of the $\mathrm{d} n / \mathrm{d} c$ values $^{27}$ (Figure S6). In the widespread cononsolvency phenomenon, preferential adsorption of one of the solvents can account for the different polymer assembly ${ }^{26}$ or phase separation ${ }^{33}$ at certain cosolvent compositions. In the present case, an analogue conjecture would be the preferential solvation of $\mathrm{PB}$ chains by the minority $g \mathrm{~PB}$ that could inhibit light-induced cluster formation in $\mathrm{CHCl}_{3}$. This conjecture certainly needs further investigation.

We are here primarily concerned with the existence and "efficiency" of patterning upon irradiation. By varying the solvent and the comonomer, we uncovered a broad range of diameters and time scales, showing the importance of the chemical environment. The observed variation of the pattern diameter led to different beam dimensions despite the same exciting beam. It is not clear to us what should define the pattern size. Drawing a parallel with nonlinear optical materials, one may expect the pattern diameter to depend on the beam dimension, the laser wavelength and power, and the nonlinearity. For example, in the case of a Kerr medium with nonlinearity $n_{2}$, the steady-state diameter of the wave-guiding pattern is determined by the coefficient of the nonlinear response and varies as $\sim \lambda\left(n_{2} I\right)^{-1 / 2}$. $^{34}$ Slower and broader patterns could therefore be the result of weaker nonlinearity, with less self-focusing.

On the basis of the presented results, we here have an integrating nonlinearity (irreversible increase of the refractive index under irradiation), possibly with saturation. Concentration gradients are expected to be unstable in polymer solutions as they will create osmotic pressure gradients that should relax in solutions. The ability to sustain such a gradient is a sign of an attractive force/energy that remains after the light is turned off. This is reminiscent of some photoreactive systems. ${ }^{18}$ There, a phenomenological nonlinear optical model was developed that captures well the time evolution of the pattern. It solved coupled equations, an evolution equation for the time/intensity dependent on the refractive index, and a nonlinear Schrodinger equation for light propagation. The solutions can be obtained numerically. ${ }^{18}$ Such a phenomenological modeling would certainly be useful in our case. It may help to understand the observed behavior. However, proper simulations will require good knowledge of the nonlinearity type that is not fully available yet. In particular, the size of the propagating beam is a transient feature, and the refractive index evolution can be calculated.

In this context, we should mention that the effect is not related to the optical trapping in aqueous solutions of various polymers near phase separation. ${ }^{35-37}$ A similar electrostriction ${ }^{13,14,38,39}$ based laser-induced phase separation was reported in a binary liquid mixture near the binodal phase region. ${ }^{40}$

These observations point toward the importance of local length scales. The observed increase of concentration, following the irradiation, has to be the consequence of locally attractive forces between polymer chains. Given the importance of the precise distribution of solvent and comonomer, one may expect that a molecular or supramolecular assembly process might be triggered by the light. Specific monomer-solvent interactions, responsible for this "clustering", are conceivably different in different solvents and comonomers. For example, the much larger dipole moment of $\mathrm{CHCl}_{3}$ compared to that of $\mathrm{C}_{6} \mathrm{H}_{12}$ will certainly affect the local monomer-solvent interaction. This different interaction between the solvent and the monomer 
possibly plays a role in both pattern size and kinetics. The solvent quality does not provide a good measure of the specifics of the interactions as the two good solvents can lead to different patterns. The change from exponential (eventually reaching saturation) to linear growth may also be a consequence of the different chemical environment.

\section{CONCLUSIONS}

The formation of fibrillar structures upon mild laser irradiation in viscous polydiene solutions in organic solvents was investigated. The involved mechanical stresses were not sufficient to activate the mechanochemical groups of $g \mathrm{~PB}$, as indicated by NMR spectroscopy. The light-induced structure formation was found to depend on the combined influence of the solvent as well the chemical nature and the relative concentration of the comonomer of the 1,4-butadiene. The qualitative differences of the formation mechanism (different pattern diameter, different kinetics type, complete pattern suppression) depend not only on the polymer repeating unit (homopolymer, copolymers) but also on the type of the solvent. Variations of solvents and comonomers affect both the pattern diameter and the refractive index contrast growth kinetics. Strikingly, for $g \mathrm{~PB}$, the solvent defined whether the light effect occurred or not. For all other cases, the light effect took place. Characterization experiments in different solvents emphasized their impact not being reflected in the solution thermodynamics $\left(A_{2}\right)$. The results clearly highlight the importance of the chemical environment in the materials response.

The laser-driven pattern formation seems to arise from a combination of a photon-triggered polymer-polymer attraction and an "assembly" process within the irradiated area. The irreversibility of the pattern indicates a long-lived assembly, possibly due to chemical cross-linking. More work on physicochemical characterization of the patterned material might reveal the nature of the assembly and possible differences with the pristine materials. We here have a new type of photoreactive material based on polymer solutions. A quantitative description of the relationship between the growth rate and the chain composition appears out of reach due to the entangled effects of solvent and comonomer ( $g \mathrm{DCC}$, styrene, 1,2-butadiene) along the PB chains. The specific properties of these materials could be of interest for lithography or waveguide-based devices.

\section{ASSOCIATED CONTENT}

\section{S Supporting Information}

The Supporting Information is available free of charge on the ACS Publications website at DOI: 10.1021/acs.jpcb.8b02463.

Description of phase contrast microscopy, phase contrast images of all irradiated samples after $300 \mathrm{~s}$, results of NMR measurements in irradiated and nonirradiated solutions of $g \mathrm{~PB}$ in $\mathrm{C}_{6} \mathrm{H}_{12}$, photon correlation spectroscopy measurements of $\mathrm{PB}$ and $g \mathrm{~PB}$ in $\mathrm{CHCl}_{3}, \mathrm{~d} n / \mathrm{d} c$ measurements in $\mathrm{PB}$ and $g \mathrm{~PB}$ in $\mathrm{CHCl}_{3}$ and $\mathrm{C}_{6} \mathrm{H}_{12}$, and extra kinetics results (PDF)

\section{AUTHOR INFORMATION}

\section{Corresponding Authors}

*E-mail: benoit@iesl.forth.gr (B.L.).

*E-mail: fytas@mpip-mainz.mpg.de (G.F.).

ORCID $\odot$

A. Bogris: 0000-0003-0328-1701

J. Wang: 0000-0002-4503-5026
M. Anyfantakis: 0000-0002-4572-5641

B. Loppinet: 0000-0003-1855-7619

S. L. Craig: 0000-0002-8810-0369

H-J. Butt: 0000-0001-5391-2618

Notes

The authors declare no competing financial interest.

\section{ACKNOWLEDGMENTS}

We acknowledge the Foundation for Research and Technology Hellas (FORTH) and Max Planck Institute for Polymer Research (MPIP) for financial support. G.F. acknowledges partial support from ERC AdG SmartPhon (No. 694977). We thank Prof. E. W. Meijer for fruitful discussions, Mrs. B. Mueller (MPIP, Mainz) for the $\mathrm{d} n / \mathrm{d} c$ measurements, and Dr. M. Kaliva (FORTH) for helping with the NMR measurements. We also thank S. Coppola for kindly providing the styrene butadiene rubber (SBR) sample.

\section{REFERENCES}

(1) Takacs, L. The Historical Development of Mechanochemistry. Chem. Soc. Rev. 2013, 42, 7649-7659.

(2) Jerca, F. A.; Jerca, V. V.; Hoogenboom, R. Photoresponsive Polymers on the Move. Chem. 2017, 3, 533-536.

(3) Hu, Y.; Li, Z.; Lan, T.; Chen, W. Photoactuators for Direct Optical-to-Mechanical Energy Conversion: From Nanocomponent Assembly to Macroscopic Deformation. Adv. Mater. 2016, 28, $10548-10556$.

(4) White, T. J.; Broer, D. J. Programmable and Adaptive Mechanics with Liquid Crystal Polymer Networks and Elastomers. Nat. Mater. 2015, 14, 1087.

(5) Chen, Y.; Spiering, A. J. H.; Karthikeyan, S.; Peters, G. W. M.; Meijer, E. W.; Sijbesma, R. P. Mechanically Induced Chemiluminescence from Polymers Incorporating a 1,2-Dioxetane Unit in the Main Chain. Nat. Chem. 2012, 4, 559-562.

(6) Klukovich, H. M.; Kouznetsova, T. B.; Kean, Z. S.; Lenhardt, J. M.; Craig, S. L. A Backbone Lever-Arm Effect Enhances Polymer Mechanochemistry. Nat. Chem. 2013, 5, 110-114.

(7) Ramirez, A. L.; Kean, Z. S.; Orlicki, J. A.; Champhekar, M.; Elsakr, S. M.; Krause, W. E.; Craig, S. L. Mechanochemical Strengthening of a Synthetic Polymer in Response to Typically Destructive Shear Forces. Nat. Chem. 2013, 5, 757-61.

(8) Clough, J. M.; Balan, A.; van Daal, T. L.; Sijbesma, R. P. Probing Force with Mechanobase-Induced Chemiluminescence. Angew. Chem., Int. Ed. 2016, 55, 1445-9.

(9) Clough, J. M.; Creton, C.; Craig, S. L.; Sijbesma, R. P. Covalent Bond Scission in the Mullins Effect of a Filled Elastomer: Real-Time Visualization with Mechanoluminescence. Adv. Funct. Mater. 2016, 26, 9063-9074.

(10) Ducrot, E.; Chen, Y.; Bulters, M.; Sijbesma, R. P.; Creton, C. Toughening Elastomers with Sacrificial Bonds and Watching Them Break. Science 2014, 344, 186-189.

(11) Bai, R.; Suo, Z. Optomechanics of Soft Materials. J. Appl. Mech. 2015, 82, 071011-071011-9.

(12) Chu, S. Laser Manipulation of Atoms and Particles. Science 1991, 253, 861-6.

(13) Guck, J.; Ananthakrishnan, R.; Moon, T.; Cunningham, C.; Käs, J. Optical Deformability of Soft Biological Dielectrics. Phys. Rev. Lett. 2000, 84, 5451-4.

(14) Casner, A.; Delville, J.-P. Giant Deformations of a LiquidLiquid Interface Induced by the Optical Radiation Pressure. Phys. Rev. Lett. 2001, 87, 054503.

(15) Shin, H.; Rakich, P. T. Optomechanics: Photons That Pivot and Shuttle. Nat. Nanotechnol. 2014, 9, 878-880.

(16) Ren, M.; Huang, J.; Cai, H.; Tsai, J. M.; Zhou, J.; Liu, Z.; Suo, Z.; Liu, A.-Q. Nano-Optomechanical Actuator and Pull-Back Instability. ACS Nano 2013, 7, 1676-1681. 
(17) Man, W.; Fardad, S.; Zhang, Z.; Prakash, J.; Lau, M.; Zhang, P.; Heinrich, M.; Christodoulides, D. N.; Chen, Z. Optical Nonlinearities and Enhanced Light Transmission in Soft-Matter Systems with Tunable Polarizabilities. Phys. Rev. Lett. 2013, 111, 218302.

(18) Biria, S.; Morim, D. R.; An Tsao, F.; Saravanamuttu, K.; Hosein, I. D. Coupling Nonlinear Optical Waves to Photoreactive and Phase-Separating Soft Matter: Current Status and Perspectives. Chaos 2017, 27, 104611.

(19) Fialkowski, M.; Bishop, K. J. M.; Klajn, R.; Smoukov, S. K.; Campbell, C. J.; Grzybowski, B. A. Principles and Implementations of Dissipative (Dynamic) Self-Assembly. J. Phys. Chem. B 2006, 110, 2482-2496.

(20) Sigel, R.; Fytas, G.; Vainos, N.; Pispas, S.; Hadjichristidis, N. Pattern Formation in Homogeneous Polymer Solutions Induced by a Continuous-Wave Visible Laser. Science 2002, 297, 67-70.

(21) Anyfantakis, M.; Loppinet, B.; Fytas, G.; Pispas, S. Optical Spatial Solitons and Modulation Instabilities in Transparent Entangled Polymer Solutions. Opt. Lett. 2008, 33, 2839-2841.

(22) Dian-Yang, L.; Ming, L.; Shu-Jie, W.; Zhi-Wei, L. LaserInduced Pattern Formation from Homogeneous Polyisoprene Solutions. Chin. Phys. B 2008, 17, 2156.

(23) Anyfantakis, M.; Königer, A.; Pispas, S.; Köhler, W.; Butt, H.-J.; Loppinet, B.; Fytas, G. Versatile Light Actuated Matter Manipulation in Transparent Non-Dilute Polymer Solutions. Soft Matter 2012, 8, 2382-2384.

(24) Loppinet, B.; Somma, E.; Vainos, N.; Fytas, G. Reversible Holographic Grating Formation in Polymer Solutions. J. Am. Chem. Soc. 2005, 127, 9678-9679.

(25) Lenhardt, J. M.; Black, A. L.; Craig, S. L. GemDichlorocyclopropanes as Abundant and Efficient Mechanophores in Polybutadiene Copolymers under Mechanical Stress. J. Am. Chem. Soc. 2009, 131, 10818-10819.

(26) Gillissen, M. A. J.; Koenigs, M. M. E.; Spiering, J. J. H.; Vekemans, J. A. J. M.; Palmans, A. R. A.; Voets, I. K.; Meijer, E. W. Triple Helix Formation in Amphiphilic Discotics: Demystifying Solvent Effects in Supramolecular Self-Assembly. J. Am. Chem. Soc. 2014, 136, 336-343.

(27) Becker, A.; Köhler, W.; Müller, B. A Scanning Michelson Interferometer for the Measurement of the Concentration and Temperature Derivative of the Refractive Index of Liquids. Ber. Bunsenges. Phys. Chem. 1995, 99, 600-608.

(28) Barty, A.; Nugent, K.; Paganin, D.; Roberts, A. Quantitative Optical Phase Microscopy. Opt. Lett. 1998, 23, 817-819.

(29) Barone-Nugent, E.; Barty, A.; Nugent, K. Quantitative PhaseAmplitude Microscopy I: Optical Microscopy. J. Microsc. 2002, 206, 194-203.

(30) Roberts, A.; Ampem-Lassen, E.; Barty, A.; Nugent, K. A.; Baxter, G. W.; Dragomir, N.; Huntington, S. Refractive-Index Profiling of Optical Fibers with Axial Symmetry by Use of Quantitative Phase Microscopy. Opt. Lett. 2002, 27, 2061-2063.

(31) Anyfantakis, M.; Pamvouxoglou, A.; Mantzaridis, C.; Pispas, S.; Butt, H. J.; Fytas, G.; Loppinet, B. Kinetics of Light-Induced Concentration Patterns in Transparent Polymer Solutions. J. Phys. Chem. B 2017, 121, 7180-7189.

(32) Khatri, C. A.; Pavlova, Y.; Green, M. M.; Morawetz, H. Chiral Solvation as a Means to Quantitatively Characterize Preferential Solvation of a Helical Polymer in Mixed Solvents. J. Am. Chem. Soc. 1997, 119, 6991-6995.

(33) Mukherji, D.; Marques, C. M.; Kremer, K. Polymer Collapse in Miscible Good Solvents Is a Generic Phenomenon Driven by Preferential Adsorption. Nat. Commun. 2014, 5, 4882.

(34) Boyd, R. W. Nonlinear Optics, 3rd ed.; Academic Press: Burlington, 2008.

(35) Borowicz, P.; Hotta, J.-i.; Sasaki, K.; Masuhara, H. LaserControlled Association of Poly(N-Vinylcarbazole) in Organic Solvents: Radiation Pressure Effect of a Focused near-Infrared Laser Beam. J. Phys. Chem. B 1997, 101, 5900-5904.

(36) Hofkens, J.; Hotta, J.; Sasaki, K.; Masuhara, H.; Iwai, K. Molecular Assembling by the Radiation Pressure of a Focused Laser
Beam: Poly(N-Isopropylacrylamide) in Aqueous Solution. Langmuir 1997, 13, 414-419.

(37) Smith, T. A.; Hotta, J.-i.; Sasaki, K.; Masuhara, H.; Itoh, Y. Photon Pressure-Induced Association of Nanometer-Sized Polymer Chains in Solution. J. Phys. Chem. B 1999, 103, 1660-1663.

(38) Conti, C.; DelRe, E. Optical Supercavitation in Soft Matter. Phys. Rev. Lett. 2010, 105, 118301.

(39) Tsori, Y. Colloquium: Phase Transitions in Polymers and Liquids in Electric Fields. Rev. Mod. Phys. 2009, 81, 1471-1494.

(40) Walton, F.; Wynne, K. Control over Phase Separation and Nucleation Using a Laser-Tweezing Potential. Nat. Chem. 2018, 10, 506. 